\title{
O QUE O PACIENTE HOSPITALIZADO SABE . SOBRE SEUS EXAMES LABORATORIAIS E COMPLEMENTARES
}

\author{
(*) Aracy Luiza Viazzolli dos Santos
}

\section{Introdução}

Motivados pela experiência de mais de uma década na docência de enfermagem médica, resolvemos estudar um aspecto da interração do paciente, o qual, sempre nos pareceu constituir problemática aparentemente sem solução - a do conhecimento que o paciente tem sobre os seus exames laboratoriais e complementares no que diz respeito à responsabilidade da enfermagem em informá-lo.

Naturalmente, espera-se que grande parcela do conhecimento sobre os exames laboratoriais e complementares seja ministrada pelo médico, já que isso constitui uma de suas atribuiçōes junto ao paciente, porém, a realidade tem-se mostrado diferente. É à enfermeira que cabe grande parte dessa tarefa delegada, que via de regra, ela também não consegue levar a efeito.

$E$ É do conhecimento de todos os profissionais da enfermagem a forma pela qual o paciente é enviado aos diversos serviços hospitalares, para ser submetido a exames dos mais variados, sem receber informação alguma senão uma solicitação lacônica do atendente do encaminhamento que lhe diz: "Sr. José, vista o seu roupão que o Sr. vai fazer um exame".

Portanto, a nossa vivência no magistério da enfermagem despertou nosso interesse no assunto e propusemo-nos a fazer uma pesquisa bibliográfica preliminar, abrangendo as duas últimas décadas da leiteratura específica, tanto nacional quanto estrangeira e averiguamos que a problemática já havia sido ligeiramente abordada no estrangeiro, associado a outros problemas de enfermagem $(1,2$,

* Professôra Assistente de Enfermagem Médica do Departamento MédicoCirúrgico da Escola de Enfermagem da USP. 
$3,4,5,6,9,11,12,13,14,15,16,17,18,23,25)$. A bibliografia. citada, forneceu-nos, então, dados sobre a existência do problema, o qual havia sido claramente identificado, exigindo porém, um estudo específico, em nosso meio.

Empregamos aproximadamente 6 (seis) meses do ano de 1972, na coleta e tabulação de dados para o trabalho.

\section{Objetivos}

Traçamos 4 (quatro) objetivos que nortearam nosso trabalho, como seguem:

1. identificar a problemática do conhecimento do paciente em relação aos seus exames laboratoriais e complementares;

2. estudar o interêsse do paciente em conhecer os exames a. que é submetido;

3. avaliar a orientação recebida pelo paciente em relação aos exames exigidos pelo médico;

4. estudar soluções viáveis para o problema.

III. Definição de Termos

\section{Conceitual}

"Exames de laboratório, são empregados como meios de ajuda específica ou complementar na confirmação ou exclusão de doenças. clinicamente suspeitadas, especialmente aquelas que apresentam problemas de confirmação de diagnóstico".

\section{Fonte de consulta}

KOLMER, I. A. O diagnóstico clínico pelos exames de laboratório.

Vol. I, Ed. Guanabara, Rio de Janeiro, 1946.

\section{Pertinente (ou especifica)}

Todos os exames, aos quais o paciente foi submetido, foram considerados indistintamente, incluindo portanto, os exames complementares e os laboratoriais, tanto para confirmação como para exclusão de diagnóstico.

\section{Metodologia}

1. A população estudada foi constituida de 120 pacientes hospitalizados em 4 (quatro) hospitais gerais, com um mínimo de 300 
leitos e um Serviço de Enfermagem organizado. A seleção da população foi ditada pelos seguintes critérios, pré-determinados:

1.1 - 30 pacientes em cada hospital (15 homens e 15 mulheres);

1.2 - pacientes de primeira internação hospitalar;

1.3 - pacientes com permanência mínima de 72 horas;

1.4 - pacientes a partir dos 15 anos de idade;

1.5 - pacientes capazes de manter uma entrevista;

1.6 - pacientes acamados e ambulantes de Clínicas Médicas, Cirúrgicas e Especialidade afins.

2. Foi usada a técnica da entrevista individual por ser considerada a mais eficaz para se estabelecer uma comunicação entre os pacientes selecionados e o entrevistadorr desconhecido $(2,3,4,5$, $20,21,22,24,25$ ) .

Elaborou-se também uma ficha-individual, abrangendo dois aspectos:

2.1 características da população (idade, sexo, escolaridade, tempo de permanência hospitalar);

2.2 formulário destinado a avaliar os seguintes dados:

- interesse em conhecer os exames;

- tipo de informante;

- época em que a informação foi recebida.

- 3. Critérios adotados para fins operacionais, a fim de poder tabular os dados obtidos:

3.1 os inúmeros exames pesquisados foram agrupados em dois grandes grupos:

- exames específicos (incluindo os exames relacionados com o diagnóstico provisório ou definitivo do paciente);

- exames inespecíficos (aqueles não relacionados com o diagnóstico provisório ou definitivo do paciente);

3.2 quanto ao tipo de informação recebida pelo paciente, decidiu-se dividí-la em 3 (três) grupos:

- satisfatória - seria a classificação da resposta que, além de informar especificamente sobre o exame e seu objetivo, ainda acrescentasse alguma orientação quanto ao preparo do paciente para o mesmo;

- pouco satisfatória - seria a informação dada ao paciente, incluindo apenas a natureza do exame. Ex.: uma esofagoscopia é a introdução de um tubo no esôfago, para o médico poder estudá-lo;

- insatisfatória - seria considerada a resposta que não desse informação alguma ao paciente, nem sobre o tipo de exame, nem sobre o seu preparo;

3.3 em relação aos informantes, decidiu-se investigar quais os elementos mais diretamente ligados ao paciente hospitalizado que 
se submete a exames, e dos quais ele poderia receber alguma informação: médico, enfermeira, auxiliar de enfrmagem, atendente, outros (englobando-se, aqui, técnicos de laboratório, colegas de enfermaria e hospital, familiares)

\section{Resultados}

Em linhas gerais os resultados obtidos, foram os seguintes:

1. Uma grande maioria, 107 pacientes, $(88,4 \%)$, manifestou interesse em conhecer os exames a que havia sido submetida, não ccorrendo diferenças numéricas, marcantes, entre homens e mulheres.

2. Uma grande maioria de pacientes era alfabetizada, 95 pacientes $(79,1 \%)$. Os analfabetos constituiram apenas $1 / 5$ da população estudada.

3. Considerando o grupo de pacientes submetidos a exames específicos, cerca de $60 \%$ (72 pacientes), não recebeu informação alguma e cerca de apenas 40\% (48 pacientes), foi informado. O pessoal de enfermagem, constituido de auxiliares de enfermagem e atendentes, informou $10 \%$ (12 pacientes) da população submetida a exames específicos, que por sua vez representa pouco mais da metade da população estudada.

4. Relacionando o tipo de informação recebida com o interesse demonstrado pelos pacientes em conhecer seus exames, foram obtidos os seguintes dados:

- do 120 pacientes estudados, menos de 10\% (12 pacientes)

foram informados de maneira satisfatória;

consideradas insatisfatórias; alguma.

\section{Conclusões}

Analisando e comparando os dados obtidos nessa pesquisa, chegamos, em linhas gerais, às seguintes conclusões:

1. a grande maioria dos pacientes submetidos a exames específicos mostrou-se interessada em obter informações sobre os mesmos;

2. o tempo de permanência média do paciente no hospital (17 dias) pode ser considerado fator favorável para ensino dos mesmos.

3. o número reduzido de analfabetos, $1 / 5$ da população, constitue mais um fator favorável para o ensino; 
4. cerca de metade dos pacientes alfabetizados, os quais representam cerca de $75 \%$ da população pesquisada, submeteu-se a exames específicos, e desses, $50 \%$ apenas, obtiveram informações sobre os mesmos;

5. dos 54 pacientes informados sobre seus exames, ccube ao médico informar 36, à enfermeira 1, ao auxiliar de enfermagem 3 , ao atendente 1 e a outros 13;

6. o único paciente informado pela enfermeira, apesar de estar preocupado com o resultado de um exame inespecífico, recebeu a resposta lacônica: "Não deu nada", sem maiores explicações.

\section{Recomendações}

De um modo geral, as recomendações giram em torno de 3 (três) fatores determinantes para a profissional de enfermagem:

- Tempo hábil para a enfermeira poder orientar;

- interesse da enfermeira em orientar;

- tipo de preparo escolar que a profissional de enfermagem recebeu sobre a importância de orientar o paciente sobre os exames a que é submetido no hospital.

Para finalizar, achamos, ainda, que cada escola deve procurai' analizar bem qual o tipo de profissional que pretende formar, ressaltando-se principalmente o fator unidade de filosofia de ensino de seu corpo docente.

\section{VIII - BIBLIOGRAFIA E REFERENCIAS BIBLIOGRÁFICAS}

1. AASTERUD, M. - Explanation to the patient. Nurs Forum, 2: 36, 1963.

2. ABDELlah, F. G. \& LEVINE, E. - Polling patients and personel: what factors affect patients opinions of their nursing care. Hospitals, 31 (16) : $61-4$, nov. 1957.

3. $\ldots \ldots \ldots \ldots \ldots \ldots \ldots \ldots \ldots$ Polling patients and personel: what hospitals have done to improve patient care. Hospitals, 31 (16): 43-4. dec. 1957.

4. $\ldots \ldots \ldots \ldots \ldots \ldots \ldots \ldots$ Polling patients and personel: what patients say about their nursing care. Hospitals, 31 (21): 44-8, 1 nov. 1957.

5. $\quad \ldots \ldots \ldots \ldots \ldots \ldots \ldots \ldots \ldots$ Polling patients and personel: what personel say about nursing care. Hospitals, 31: 53-7. 1 dec. 1957.

6. BERMOSK, L. S. - Interviwing: a key to therapeutic communication in nursing pratice. Nurs Clin Norte Am, 1 (2): 205-14, jun. 1965.

7. BURTON, G. - Nurse and patient: the influence or human relationships. London. Tavistock, 1958.

8. ................ Personal, impersonal. and interpersonal relation a guide for nurses. 2nd ed. New York, Springer, 1964. 
9. CARNER, D. C. - You can manage people better if you tell them more. Mod Hosp, 109: 96, sep. 1967.

10. GRIFFITHS, D. E. - Teoria da Administração Escolar. S. Paulo, Companhia Editora Nacional, Ed. da USP, 1971.

11. DICHTER, E. - A psychological study of the hospital-patient relationship: what the patient really wants from the hospital. Mod Hosp, 83 (3): $51-4$, sep. 1954.

12. $\ldots \ldots \ldots \ldots \ldots \ldots \ldots$ A psychological study of the hospital-patient relationsrip: the patient's greatest need is security. Mod Hop. 83 (4): 56-8, oct. 1954.

13. ............... A psychological study of the hospital-patient relationship: how "secure" is your hospital. Mod Hosp, 83 (5): 61-3, nov. 1954.

14. DICHTER, E. - A psychological study of the hospital-patient relationship: what the community trinks of the hospital. Mod Hosp, 83 (6) : 69-71, dec. 1954 .

15. ................. A psychological study of the hospital-patient relationship: what the community thinks of the hospital. Mod Hosp. 84 (1): 74-6, jan. 1955.

16. $\ldots \ldots \ldots \ldots \ldots \ldots$ A psychological study of the hospital-patient relationship: the administrator sets the tone. Mod Hosp, 84 (2): 59-63, feb. 1955.

17. JOURARD, S. M. - How well do you know you patient? Am $J$ Nurs. 59 (11): 1568-71, nov. 1959.

18. KAMIYAMA, Y. - O doente rospitalizado e sua percepção quanto a prioridade de seus problemas. São Paulo, 1972. Tese-Escola de Enfermagem da Universidade de São Paulo.

19. KOLMER, I. A. - O diagnóstico clínico pelos exames laboratoriais. Vol. I, Ed. Guanabara, Rio de Janeiro. 1946.

20. PAYNE, S. S. - The art of asking questions. 5th ed. Princeton, New Jersey. 1951.

21. PEPLAU, H. E. - Professional closeness ... as a special kind of involment with a patient, client or family group. Nurs Forum, 8 (4): 342-60. 1969.

22. $\ldots \ldots \ldots \ldots \ldots \ldots$ Talking with patients. Am J Nurs. 60 (7):

23. SKIPER Jr., J. K. et al - Some barriers to communication between patients and hospital functionaries. Nurs. Forum. 2 (1): 15-23, 1963.

24. $\ldots \ldots \ldots \ldots \ldots \ldots \ldots \ldots$ Some possible consequences of limited communication between patients and hospital functionaries. $J$ Hlth Hum Behav, 5: 34-9, 1964.

25. SKIPPER Jr.. J. K. \& R. C. - Communication and patient care. Can Nurse, 61 (7): 562f-g-h. jul, 1965. 